\title{
El efecto de la digitalización en la financiación de los medios en lenguas minoritarias: diez casos europeos
}

\author{
Iñaki Zabaleta-Urkiola ${ }^{1}$ \\ Carme Ferré-Pavia ${ }^{2}$ \\ Itxaso Fernández-Astobiza ${ }^{3}$ \\ Santiago Urrutia-Izaguirre ${ }^{4}$ \\ Arantza Gutiérrez-Paz \\ Nicolás Xamardo-González ${ }^{6}$
}

Recibido: 2014-07-01

Enviado a pares: 2014-07-01
Aprobado por pares: 2014-09-17

Aceptado: 2014-10-21

DOI: 10.5294/pacla.2015.18.1.6

Para citar este artículo / To reference this article / Para citar este artigo

Zabaleta-Urkiola, I., et al. Marzo de 2015. El efecto de la digitalización en la financiación de los medios en lenguas minoritarias: diez casos europeos. Palabra Clave 18(1), 131-155.

DOI: 10.5294/pacla.2015.18.1.6

\section{Resumen}

En un contexto en que el estudio de los medios en lenguas minoritarias, minorizadas o indígenas tiene ya un amplio recorrido, su proceso de digitalización ha sido aún poco estudiado. Esta investigación aborda cómo se han percibido los cambios que la digitalización imprime en la financiación de los medios de comunidades de lenguas minoritarias europeas. Se trata de un análisis transnacional que alcanza diez comunidades lingüísticas

\footnotetext{
Universidad del País Vasco, España. inaki.zabaleta@ehu.es

Universidad Autónoma de Barcelona, España. carme.ferre@uab.cat

Universidad del País Vasco, España. itxaso.fernandez@ehu.es

Universidad del País Vasco, España. santi.urrutia@ehu.es

Universidad del País Vasco, España. mirenanrantza.gutierrez@ehu.es

Universidad del País Vasco, España. nicolas.xamardo@ehu.es
} 
europeas: vasca, catalana, gallega, corsa, bretona, frisia, gaélico-escocesa, galesa, irlandesa y sami, con una población de hablantes global en torno a los 12 millones de personas. Se ha seleccionado una muestra representativa de 144 medios tradicionales de prensa, radio y televisión, analizados a través de cuestionarios en los que se entrevista a los responsables de estos medios, en que responden sobre los efectos de la conversión digital sobre su financiación y valoran estos cambios. Los resultados confirman que la financiación se percibe mayoritariamente como el aspecto más negativo en el que ha influido la migración digital, aunque es destacable la incapacidad de dar respuestas concretas sobre estos efectos por parte de un número importante de los gestores encuestados. Estos destacan otras áreas productivas donde el efecto es considerado, en cambio, positivo: casi el $70 \%$ opina que la digitalización tiene un efecto favorable sobre los contenidos y el $77 \%$ que también es benéfico para la audiencia. Los datos cuantitativos se combinan con la parte cualitativa del relato de cada comunidad.

\section{Palabras clave}

Financiación, digitalización, crisis financiera en los medios, medios en lenguas minoritarias, percepción de la crisis (Fuente: Tesauro de la Unesco). 


\section{The Effect of Digitalization on the Funding of the Media in Minority Language: Ten European Cases}

\section{Abstract}

In a context in which the study of the media in minority languages, whether minoritized or indigenous, has a long story already - the digitalization process has not yet been widely studied. This study addresses how the changes imprinted by digitization have been perceived on the funding of the media of European communities of minority languages. This is a transnational analysis that encompasses ten European language communities: Basque, Catalan, Galician, Corsican, Breton, Frisian, Gaelic-Scottish, Welsh, Irish and Sami, with a global population of speakers of around 12 million individuals. A representative sample of 144 traditional media of press, radio and television were selected, which were then analyzed by way of questionnaires used to interview the individuals responsible for these media, in which they respond regarding the effects of the digital conversion on their funding and where they evaluate these changes. The findings confirm that the funding is largely perceived as the most negative aspect that has been influenced by the digital migration, although it is necessary to highlight the inability to provide clear answers on these effects by a significant number of managers interviewed. However, they also highlight other productive areas where the effect is being considered as being positive: nearly $70 \%$ believe that digitization has a favorable effect on the contents, and $77 \%$ that it also is beneficial to the audience. The quantitative data was combined with the qualitative part of the statements given by each community.

\section{Keywords}

Funding, digitalization, financial crisis of the media, media in minority languages, perception of crisis (Source: UNESCO Thesaurus). 


\section{0 efeito da digitalização no financiamento dos meios de comunicação em línguas minoritárias: dez casos europeus}

\section{Resumo}

Em um contexto em que o estudo dos meios de comunicação em línguas minoritárias, menorizadas ou indígenas já tem uma ampla trajetória, seu processo de digitalização ainda foi pouco estudado. Esta pesquisa aborda como foram percebidas as mudanças que a digitalização imprime no financiamento dos meios de comunicação de comunidades de línguas minoritárias europeias. Trata-se de uma análise transnacional que abrange dez comunidades linguísticas europeias: vasca, catalã, galega, corsa, bretã, frísia, gaélico-escocesa, galesa, irlandesa e sami, com uma população de falantes global em torno de 12 milhões de pessoas. Foi selecionada uma amostra representativa de 144 meios de comunicação tradicionais da imprensa, rádio e televisão, analisados através de questionários nos quais se entrevista aos responsáveis desses meios de comunicação, em que respondem sobre os efeitos da conversão digital sobre seu financiamento e avaliam essas mudanças. Os resultados confirmam que o financiamento é percebido majoritariamente como o aspecto mais negativo em que a migração digital influenciou, ainda que seja notável a incapacidade de dar respostas claras sobre esses efeitos por parte de um número importante dos gestores entrevistados. Estes destacam outras áreas produtivas em que o efeito é considerado, no entanto, positivo: quase $70 \%$ opinam que a digitalização tem um efeito favorável sobre os conteúdos e $77 \%$ que também é benéfico para o público. Os dados quantitativos combinam com a parte qualitativa do relato de cada comunidade.

\section{Palavras-chave}

Financiamento, digitalização, crise financeira nos meios de comunicação, meios de comunicação em línguas minoritárias, percepção da crise (Fonte: Tesauro da Unesco). 


\section{Introducción}

Medios de todo el mundo siguen buscando el modelo de financiación que los aparte de la crisis y les genere los beneficios de antaño, con ensayos que incluyen o no el pago, la suscripción o la creación de comunidades de lectores. El proceso de digitalización de los últimos 20 años (despliegue de medios y plataformas digitales, tecnología, canales digitales de medios tradicionales y cambio de formas de consumo, entre otras realidades), forma parte del problema tanto como de la solución. En el campo de las lenguas minoritarias, sus retos pueden ser aún más importantes, en tanto no siempre están soportadas por un mercado fuerte que las apoye. Esta investigación se plantea qué áreas de negocio y producción se han percibido como las más afectadas por la digitalización, en qué sentido está alterada la financiación y si el proceso, en su globalidad, se vive como algo positivo o negativo en las empresas europeas de estas comunidades. En el caso de las diez de este estudio, la transición de la emisión analógica a la digital se convirtió en una obligación tecnológica y reguladora que generó incertidumbre. En 2009, en las diez comunidades lingüísticas estudiadas el 29\% de los medios tradicionales todavía no tenían ningún tipo de sitio web, el $47 \%$ tenía un sitio corporativo básico sobre el medio de comunicación y sólo el $24 \%$ tenía sitios web con servicios actualizados de información (Zabaleta et al., 2013). Si tomamos el caso latinoamericano, las cifras pueden variar, teniendo en cuenta como ejemplo que en 2009 más del 70\% de las radios indígenas colombianas no tenían ni acceso a Internet (ONIC, 2009, citado en Cuesta, 2012).

En Europa, la intensificación de la digitalización ha coincidido con la crisis financiera desde 2007 y su conversión en crisis económica profunda a partir de 2008 (WAN-IFRA, 2011). La situación se ha podido agravar en los medios en lenguas minoritarias por su situación de debilidad, y esta investigación se ha aproximado a los medios para que sean ellos mismos los que narren sus dificultades y establecer a nivel europeo una comparativa de sus experiencias.

El interés de tomar como marco las lenguas en sus contextos culturales y no estatales, en un estudio sobre la dificultad profesional para afrontar 
la digitalización, reivindica este espacio que responde a la cultura y debe ser analizado en sus retos más contemporáneos.

Los estudios sobre los procesos de digitalización han sido tradicionalmente considerados en un contexto estatal o de nación-estado, una unidad de referencia que puede generar otras subcategorías (regional, internacional, etc.). Esta perspectiva sigue la teoría del estatismo (Taylor, 1996) o nacionalismo metodológico (Wimmer \& Glick Schiller, 2002, 2003), que aborda los fenómenos circunscritos en la nación-estado. Académicos de diferentes campos han criticado especialmente esta teoría, aduciendo que oscurece la diversidad cultural y los procesos de transnacionalización y globalización (Amelina, Nergiz, Faist \& Glick Schiller, 2012), elude las conexiones y prácticas transculturales (Meinhof \& Triandafyllidou, 2006) y privilegia la homogeneidad cultural (Robins, 2006).

\section{Marco teórico-referencial}

\section{Estudios sobre medios en lenguas minoritarias}

En las dos últimas décadas, la investigación académica sobre las lenguas minoritarias se ha incrementado notablemente, con la particularidad de que la mayoría de estudios se centran en una sola comunidad. Existen algunos estudios más plurales, como el trabajo de Riggins (1992) sobre la consecución de un espacio mediático hecho por indígenas e inmigrantes de una manera que integre minorías; sobre modelos de medios para la supervivencia aborigen (Browne, 1996; Wilson, Stewart \& Córdova, 2008; Alia, 2010); la publicación de Hogan-Brun y Holff (2003) sobre los contextos, estatus y prospectiva de las lenguas minoritarias en Europa; los estudios de Kirk y Baoill (2003) dedicados al sistema de medios en lenguas minoritarias de Irlanda, Irlanda del Norte y Escocia; el libro de Cormack y Houringan (2007) como un intento de definir y desarrollar el campo de los medios en lenguas minoritarias como un terreno de estudio específico, y la sugerente reflexión de McGonagle y Moring (2012) sobre el examen presente de los medios y las minorías. 
Las investigaciones desde una perspectiva estrictamente comparativa son más escasas, aunque existen sobre las funciones lingüísticas de los periodistas (Husband, 2005; Zabaleta et al., 2010a, 2013) o sobre las políticas regionales para medios de minorías e inmigrantes (Extra \& Gorter, 2008). La compilación de Jones y Uribe-Jongbloed (2013) plantea una visión global en el marco de la convergencia, la existencia de redes sociales y la capacidad de participación de las audiencias, con estudios de caso sobre comunidades concretas.

Algunos estudios sobre el avance de Internet en comunidades lingüísticas o el impacto económico de las industrias culturales tienen a los medios como un aspecto troncal pero no único de investigación en las comunidades de lenguas minoritarias (Cunliffe, 2009; Chalmers et al., 2013).

En el caso de América Latina, la realidad de las lenguas llamadas minoritarias (a veces por no ser oficiales, independientemente de su número de hablantes), refuerza una perspectiva indigenista permeada de abordes culturales, de interpretación sobre la cosmovisión de las comunidades y los retos para crear medios que las articulen. Es importante el acervo de estudios sobre la radiodifusión indígena en México (Ramos, 2009), Colombia (Cuesta, 2012), Chile (Lara \& Vera, 2003), Brasil (Iriarte, 2006) y Bolivia (Laime, 2009). Destaca especialmente en Colombia el trabajo de UribeJongbloed sobre medios en lenguas comunitarias tanto desde el foco nacional como desde una perspectiva comparativa entre las políticas de Europa y América Latina $(2007,2014)$.

\section{Digitalización y financiación}

Nos encontramos abordando en las comunidades de lenguas minoritarias en Europa un binomio de digitalización-financiación con múltiples dimensiones (Díaz Nosty, 2011). La concurrencia de ambos procesos ha causado grandes problemas en la financiación y en el modelo de negocio de las empresas periodísticas: tanto en los medios tradicionales como en los digitales las consecuencias han sido el despido de empleados, la disminución de la publicidad y bajadas de audiencia, entre otros efectos negativos (Cowan \& Whestpal, 2010). En los cibermedios, la mayor dificultad parece residir 
en la inexperiencia en el modelo de negocio adecuado al mundo digital, la necesidad de formación y el establecimiento de nuevos procesos de gestión y producción. Pese a la multidimensionalidad de la conjunción entre la financiación de los medios y su proceso de digitalización, no es fácil encontrar estudios sobre estos aspectos vinculados, y menos aún si se trata de investigaciones de casos prácticos (Dänzler, 2011; Nedeljković, 2011).

Oriella PR Network, un pool de quince agencias de comunicación independientes del mundo, publicó en su informe anual Digital Journalism una comparativa entre la percepción de 2009 y 2010 de los profesionales. ${ }^{7}$ Digitalización y crisis habían actuado al unísono para reducir el número de publicaciones, incrementando las horas de trabajo y aumentando las exigencias de contenido (Oriella, 2011; Starr, 2012). Desde un punto de vista mundial, en 2009 se sufrió un recorte del 11,4\% en el mercado global de la prensa y de un 19,1\% de la publicidad impresa. El gasto total en revistas de consumo en todo el mundo cayó un 10,6\% y el crecimiento del gasto global en Internet se frenó hasta el 8,2\% (PwC, 2010). La Asociación de la Prensa de Madrid, que cifra los medios cerrados en el periodo 2008-2013 en 284 para el caso español, no discrimina lengua ni difusión: "De los al menos 284 medios desaparecidos desde que comenzó la crisis, 182 fueron revistas, 31 diarios, 29 televisiones, 20 medios digitales, 11 publicaciones gratuitas, 9 radios y 2 agencias” (Asociación de la Prensa de Madrid, 2013).

Conjugar las audiencias locales con la competitividad en la oferta de servicios y contenidos parecía ser el reto en un contexto de adaptación tecnológica (Casero, 2010) y la reconversión del tipo de negocio hacia la creación de aplicaciones se cerró en 2011 con notas optimistas (Oriella, 2011). Según algunos autores, fue la explosión de los dispositivos móviles y la dificultad de reconvertir el negocio hacia la oferta de servicios lo que puso a los medios, entre 2009 y 2011, en un brete difícil (Fombona et al., 2012; Scolari, 2013), aunque no hay datos disgregados para la información de proximidad o de comunidades pequeñas.

7 Entre 2008 y 2010, Oriella PR Network realizó un estudio con más de 350 periodistas europeos para determinar qué impacto tuvo sobre el mundo del periodismo la llegada de Internet y la disponibilidad del ancho de banda. 
El cierre de algunos medios en lenguas minoritarias relevantes, como en el caso de España y otros, ha tenido que ver en los últimos tiempos con el descenso o la cancelación de las ayudas oficiales, o bien las decisiones de agencias y gobiernos regionales para clausurar canales públicos (Col-legi de Periodistes, 2013).

En cuanto a la digitalización de los medios en lenguas minoritarias en Europa, una investigación del equipo firmante (Zabaleta et al., 2010b) analizó el grado de extensión de la señal digital de RTV y la presencia en Internet de los medios tradicionales. Se concluyó que se ha dado una diferente velocidad de la Europa de las lenguas minoritarias en ese terreno. En algunas comunidades la digitalización no supuso un aumento ni disminución de canales de radiotelevisión (galesa, irlandesa, frisia, sami); en otras, la televisión digital obligó a que canales ya existentes aunaran fuerzas para seguir funcionando, lo que redujo el número de emisoras en lengua propia (gallega, catalana). En un tercer caso, significó el nacimiento de nuevos canales (vasca, gaélico-escocesa), y en las comunidades lingüísticas bretona y corsa no se había culminado el proceso de digitalización. ${ }^{8}$ Para el caso gaélico-escocés, Cunliffe (2009) confirma que el impulso digital en esa comunidad fue a partir de 2005.

En cuanto a la radio digital, aunque las emisoras más grandes estaban preparadas para dar el salto, las de lengua propia podían quedar silenciadas, sin acceso al nuevo dial, lo que incidiría sobre todo en las existentes en las comunidades vasca, gallega, catalana, bretona y corsa. Sin embargo, por el momento siguen emitiendo en analógico, y muchas de ellas se plantean su acceso a la red.

\section{Estructura de medios en las diez comunidades lingüísticas ${ }^{9}$}

Comunidad catalana. En esta comunidad (7,5 millones de hablantes potenciales entre 12 millones de habitantes) existen 796 empresas de medios

8 Los medios concretos, cuya cita excedería la extensión de este artículo, pueden ser consultados en Zabaleta et al. (2010b).

9 Se trata de datos de investigaciones propias del equipo para retratar la dimensión de cada comunidad. Ver Zabaleta et al. $(2008,2014)$. El censo de medios se obtiene de la investigación propia en cada país, con múltiples fuentes como instituciones, entrevistas, visitas a los mismos medios, seguimiento de nacimientos y muertes de medios y contacto con asociaciones. 
monolingües. Se cuentan alrededor de 4000 periodistas ocupados en todos estos medios.

Comunidad vasca. Para 860000 hablantes de los tres millones de población, existen 108 empresas de medios monolingües, de los cuales ocho son relevantes. Se estima que hay alrededor de 650 periodistas trabajando en los media citados.

Comunidad galesa. Situada en el Reino Unido, en Gales unas 600000 personas (tres millones de habitantes) se consideran hablantes de galés. Hay 76 empresas de medios monolingües, de las cuales cinco son relevantes. ${ }^{10} \mathrm{Un}$ centenar de periodistas trabajan a tiempo completo en los referidos medios.

Comunidad gallega. Cuenta con una población de 2,5 millones de hablantes, el 91\% de los 2,8 millones de habitantes de Galicia. El gallego cuenta con 45 empresas de comunicación monolingües y tres medios relevantes. En total, hay 464 periodistas trabajando en todos estos medios.

Comunidad irlandesa. Situada en la isla de Irlanda, se halla dividida entre la República de Irlanda, que constituye un estado, e Irlanda del Norte, parte del Reino Unido. Tiene una comunidad hablante de 600000 personas entre 2,6 millones de ciudadanos. La comunidad irlandesa cuenta con 12 empresas monolingües y cuatro medios relevantes. Unos 120 periodistas trabajan en medios de lengua irlandesa.

Comunidad bretona. La comunidad lingüística bretona está situada en Bretaña (Francia) y cuenta con 202000 bretonantes de los tres millones de habitantes. Pueden consumir 11 medios monolingües, ninguno de ellos relevantes. Unos 50 periodistas están dedicados a estos medios.

Comunidad frisia. Está situada en Frisia, provincia de Holanda. Una pequeña minoría se localiza en el norte de Alemania, en la Baja Sajonia. Al-

10 Se consideran medios relevantes todos aquellos de difusión general en la comunidad, de contenido general y periodicidad diaria o bien multisemanal para la prensa. 
rededor de 480000 personas se consideran frisioparlantes entre 650000 . El frisio es cooficial junto con el holandés. Encontramos diez empresas de comunicación monolingües, con un único medio relevante. Hay unos 80 periodistas que trabajan en medios en lengua frisia, mayoritariamente en radiotelevisión.

Comunidad sami. Situada en el norte de Escandinavia y en la península rusa de Kola, cuenta con una población que habla la lengua en torno a 21 000-30 000 personas, entre una población que no pasa de 100000 . El sami es únicamente oficial en Finlandia y en cada uno de los parlamentos sami de los tres países escandinavos. Hay tres empresas monolingües en sami nórdico. El único medio relevante es un diario privado de difusión general.

Comunidad corsa. Está situada en la isla de Córcega (Francia) y tiene 280000 habitantes, de los que unos 90000 hablan corso, además de los 45000 hablantes en el resto del Estado francés. De los tres medios monolingües ninguno se puede considerar relevante y en total se calcula que trabajan unos 28 periodistas.

Comunidad gaélico-escocesa. Situada en Escocia, forma parte del Reino Unido y tiene una población de 60000 hablantes entre cinco millones de personas. A finales de 2008 había tres medios monolingües en gaélicoescocés, todos relevantes. Se estima que hay unos 40 periodistas trabajando a tiempo completo, casi todos ellos en radiotelevisión.

\section{Cuestiones de investigación}

El objetivo general de la investigación sobre cómo ha afectado a la financiación de los medios de lenguas minoritarias el proceso de digitalización se plasma en dos preguntas de investigación básicas:

1. Cuál es la percepción de los gestores de los medios de las diez lenguas minoritarias estudiadas sobre los efectos de la digitalización en su sistema de financiación.

2. Qué evaluación hacen de este proceso en términos positivos o negativos para el medio. 


\section{Diseño metodológico}

Unos 12 millones de hablantes se comunican en lengua vasca, catalana, gallega, corsa, bretona, frisia, gaélico-escocesa, galesa, irlandesa y sami, las lenguas observadas, y en esas comunidades viven unos 30 millones de habitantes. El estudio se ha centrado en los medios tradicionales (prensa, radio y televisión) con contenido en lengua propia igual o superior al 70\%, con una frecuencia de publicación superior a un número/año, de información general o especializada y con una difusión general o local.

Se elaboró una muestra de 144 medios, pertenecientes a las diez comunidades. ${ }^{11}$ En el caso de la bretona, corsa, frisia, irlandesa, gaélico-escocesa y sami, debido al reducido número de medios monolingües, se empleó prácticamente todo el censo de medios, por lo que no hay potencial error muestral. En cambio, en las comunidades catalana, vasca, gallega y galesa se diseñó una muestra estratificada y de selección aleatoria, teniendo en cuenta tipo de medio, propiedad y difusión. De esta forma, en la muestra hay 40 medios en lengua catalana, 30 en vasca, 22 en gallega y 15 en galesa. ${ }^{12}$

Por tanto, a nivel europeo, la distribución de la muestra reflejó con importante exactitud la población total de medios, siendo la diferencia inferior al $8 \%$ en las variables de tipo de medio y propiedad. Con respecto a la variable de difusión, se consideró metodológicamente más acertado establecer dos grupos de similar tamaño, ya que el censo de medios mostraba una gran diferencia entre el número de medios locales y de difusión general. Así, la muestra final incluyó 78 medios locales y 66 de distribución general.

La herramienta metodológica aplicada fue un cuestionario cumplimentado por los responsables financieros o altos cargos de los medios. El periodo de encuesta fue en 2012, esta se hizo presencial en parte de los casos, y vía correo electrónico en otros, con las mismas preguntas. Se mandó

11 Los sistemas de medios en los diez idiomas sumaban 1032 empresas periodísticas: $52,6 \%$ en prensa, 33,8\% en radio y $13,6 \%$ en TV. Con respecto a la variable de propiedad, $38,9 \%$ eran públicos, $31,5 \%$ privados y $29,6 \%$ sociales. En cuanto a la difusión, el 77,8\% eran locales y el 22,2\% de alcance general (Zabaleta et al., 2014).

12 La muestra gallega es superior en número a la galesa porque el universo de medios de la primera es más variado en tipología que el de la segunda (con mayoría de medios impresos locales). 
por correo el mismo cuestionario a los medios que en la ronda de visitas no fueron entrevistados por falta de coincidencia de agenda.

El cuestionario consta de nueve preguntas (cinco abiertas y cuatro cerradas). Se creó un sistema de categorías que permitiera una interpretación que combinara resultados cuantitativos y cualitativos. El investigador principal llevó a cabo un estudio piloto de las respuestas y desarrolló una serie de subcategorías (financiación difícil, reducción de publicidad, reducción de ventas, reducción de costes, aumento de publicidad, pérdida/ aumento de audiencia, dificultad tecnológica, más visibilidad medio, etc.) que fueron agrupadas en un sistema de categorías más compacto y reducido (financiación, contenido, audiencia, idioma, no cambios, no sabe), más la adicional de "no contesta".

Un segundo investigador, de modo independiente, recodificó los casos del estudio piloto y revisó las definiciones de las subcategorías y categorías. En todo este proceso de pilotaje, codificación duplicada y ajuste, la fiabilidad de los intercodificadores fue superior al 95\%. Las respuestas de los informantes se recodificaron con ajustes, agrupadas en dos metacategorías: efectos directos e indirectos. Los efectos directos de la digitalización en la financiación pueden ser: 1) reducción o aumento en la publicidad, 2) reducción de ventas o 3) dificultades de financiación y efectos en la misma. Los efectos indirectos se establecen sobre la audiencia, los contenidos y el medio, el idioma y la comunidad lingüística.

\section{Resultados}

Los resultados del efecto de la digitalización (ED en adelante) sobre la financiación se presentan desde dos dimensiones: la europea y la intercomunitaria. El análisis europeo se construye a partir del total de las diez comunidades lingüísticas pero la comparación intercomunitaria permite observar las diferencias o similitudes que existen entre ellas. También la presentación de datos combina los resultados estadísticos (para la muestra general de los 144 medios) y los aspectos cualitativos de las declaraciones de los entrevistados en la comparativa intercomunitaria. 


\section{Efectos de la digitalización en la financiación}

A nivel europeo, la primera e inquietante cuestión es la dificultad de valorar qué efectos ha supuesto su proceso de migración digital. El 40\% (62 respuestas) está en la categoría de "no sabe o no contesta", lo que se puede interpretar en el sentido de que el tema para unos es desconocido, para otros provoca incertidumbre, o todavía no han tenido una experiencia práctica de gestión empresarial en el entorno digital. Parte de los encuestados, si bien son directores, editores o directores económicos de los medios, desconocen o no se atreven a opinar sobre los efectos de la digitalización en la financiación de estos. Por ejemplo, ninguno de los pocos medios en gaélicoescocés comenta nada al respecto. Hay que tener en cuenta que la encuesta presencial ya anulaba el caso de la duda ante la pregunta, por la capacidad de precisión y corrección que el contacto aportaba.

El32\% (48) de las respuestas recibidas se refieren directamente al ED en la financiación. ${ }^{13}$ El 28\% (43) destaca el ED en otras áreas, tales como el contenido ( $40 \%, 17$ respuestas), la audiencia $(33 \%, 14)$ y el idioma ( $16 \%)$. Incluso hay algunas pocas respuestas (10\%), pertenecientes a medios en lengua vasca y gallega, que indican que no hay cambios con la digitalización, bien porque no se considera que vaya a influir en el proceso productivo o bien porque aún no se ve que los medios digitales tengan credibilidad para acaparar contratos publicitarios.

A nivel intercomunitario, podemos afirmar que la proporción de respuestas sobre el ED en la financiación es similar en prácticamente todas las comunidades al valor general europeo establecido anteriormente, esto es, que uno de cada tres informantes reconoce en la financiación un ámbito afectado. La única salvedad está en los medios en lengua frisia, donde una de las cuatro respuestas ha señalado el efecto sobre la financiación y las otras tres se interesan por el ED en otros ámbitos (contenidos, audiencia e idioma). De todas formas, conviene recordar que el sistema de medios monolingües en esta comunidad es muy pequeño; igual, por lo tanto, que el número de respuestas.

13 El 100\% del total responde a más del total de encuestas porque hay cuestionarios donde se marca más de una opción. 
De modo correlativo, la proporción de respuestas referidas a "otros" ámbitos donde la digitalización tiene efecto ha sido similar en casi todas las comunidades, en torno a una de cada cuatro respuestas.

\section{Percepción sobre la financiación}

El 78\% (38) de las respuestas directamente relacionadas sobre el ED en la financiación indican que este se percibe como negativo por diversas razones: una financiación difícil, la reducción de la publicidad y/o la reducción de ventas. El editor de un semanario galés ${ }^{14}$ habla de un proceso al que son "forzados": "Todos han sido forzados a operar digitalmente pero los aspectos económicos son difíciles".

Cecille Goualle, directora de la radio local de Bretaña Radio Bro Gwened, destaca, asimismo, las dificultades financieras si no hay ayudas: "Si no se aporta ninguna ayuda específica será muy difícil invertir". La directora de antena de una radio de Córcega teme: "Habrá que tomar la ruta de la adaptación. Pero será un cambio muy difícil de sostener financieramente".

Otros encuestados destacan más la dificultad para conseguir publicidad. El editor de un medio galés apunta: "La gente espera tener información en la web gratuitamente o a través de lo que pague la publicidad. Es difícil tener anunciantes en webs galesas. Personalmente, no desearía ver anuncios en ingles en la web galesa".

En cambio, una cita de la comunidad catalana puede mostrar dualidad en la valoración sobre los efectos de la digitalización. Enric Marín, expresidente de la Corporación Catalana de Medios Audiovisuales, el ente regulador regional del audiovisual, sostiene: "La diversificación digital también puede recompensarse con nuevas formas de ingresos. Las inversiones necesarias para llevarla a cabo no siempre están al alcance de todos los medios. Si se pueden aplicar, suponen, al menos, nuevas vías para llegar a la audiencia, y por tanto, para obtener nuevos ingresos". Es decir, se valora positivamente pero se tienen en cuenta los retos. En el mismo sentido opina

14 No revelamos el nombre ni citamos el del medio cuando el entrevistado se ha acogido a la cláusula de confidencialidad. Si no, se comenta libremente. 
el consejero de un diario vasco: "Los medios digitales abarataran algunos procesos, pero los medios convencionales deberán cambiar su concepto de negocio. Vienen muchos cambios y oportunidades nuevas. No está claro todavía quién saldrá ganando y quién perderá".

Otro caso de planteamientos paradójicos sería el que propone el director de un semanario juvenil sami, quien denuncia: "La juventud sami está muy orientada a las tecnologías y las noticias online, pero todo está en noruego o en inglés". Es decir, el entorno de las tecnologías digitales favorece la incorporación de nuevos lectores pero las dificultades lingüísticas no se superan sólo con tecnología. Para el caso gallego, se indica que "no debería influir negativamente", ya que los jóvenes están preparados para ese cambio, tanto tecnológica como idiomáticamente (Caetano Díaz, director del diario Galicia Hoxe).

En cambio, el 22\% (11) de las respuestas indican que la digitalización será positiva por las siguientes razones: la ampliación de la publicidad, la reducción de costes y la potenciación de la financiación. Este sería el caso de la opinión del coordinador de proyecto de un semanario irlandés: "La digitalización y los medios digitales facilitan el acceso y la producción de contenidos y serán más baratos de producir. En veinte años se leerá online y no habrá costos del papel, que son enormes en los medios impresos. La gente necesita más y más noticias, contenidos, los lectores son insaciables".

Desde el punto de vista de la comparativa intercomunitaria, se puede concluir que la proporción de respuestas que afirman que el ED en la financiación será negativo es mayoritaria en casi todas las comunidades: alrededor de tres de cada cuatro opina de la misma manera. La única excepción la encontramos en los medios en lengua irlandesa, donde tres de cada cuatro opinan que será positivo. Por ejemplo, Mary Uí Chadhain, directora financiera de la televisión irlandesa TG4, indica: "La transformación digital será un desafío todavía mayor para los medios minoritarios. Debido a su pequeña dimensión y flexibilidad, los medios en lenguas minoritarias se pueden reposicionar bien y entrar en la tecnología digital, lo que ayudará a crear mayor y más global distribución y nuevos ingresos". 


\section{Más allá de la financiación}

$\mathrm{Al}$ abordar la segunda cuestión de investigación, observamos que el 69\% de los participantes opinan que el ED en los contenidos del medio será positivo. La razón aducida en este caso es la facilidad de producción en el entorno digital, con énfasis en la combinación del digital con el papel. En este sentido, el editor de un semanario de difusión general frisio plantea: "Creo que la combinación de publicación impresa y digital será exitosa”. Lo mismo indica Jaume Reixach, editor del semanario catalán El Triangle: "Creemos que son compatibles la prensa en papel (reportajes de investigación, artículos de opinión y entrevistas) con la digital (información de actualidad)”.

El 31\% opina que el ED en los contenidos será negativo y esto se atribuye a la dificultad tecnológica, lo que se traduce en que la debilidad de los sistemas comunicativos (medios pequeños, pocos recursos) no permite la extensión digital ni la competencia necesaria. La general manager de una radio sami lo indica así: "Casi ninguna radio minoritaria en nuestra región puede tener distribución digital en DAB (Digital Audio Broadcasting); es demasiado caro. Ni las radios locales grandes noruegas (lengua mayoritaria del estado donde están los sami noruegos) lo quieren. La digitalización es una estrategia de las grandes radios que traerá la liquidación de las radios pequeñas”. Elisabet Soldevila, directora del semanario catalán Torelló, también señala los costos como un punto de afectación en los contenidos: "La transformación digital es un proceso caro y que necesita de personal especializado, por tanto, para un periódico pequeño como el nuestro, es difícil hacer una apuesta en este sentido".

Desde un punto de vista comparativo por comunidades, la percepción de que la digitalización es positiva para la producción de contenidos se ha plasmado en las respuestas de los medios en catalán, vasco y frisio.

En cuanto a otras áreas afectadas, el 77\% opina que el ED en la audiencia del medio será positivo. Pero ello no quiere decir necesariamente que habrá más ingresos económicos, porque bastantes consideran que hay poca publicidad o pocos ingresos por publicidad en Internet. El 23\% opina que el ED en la audiencia del medio será negativo. Pese a la previsión de 
ya haber implementado plataformas digitales, en algunos medios de ámbito local no saben cómo hacer migrar a sus lectores a soportes web.

En la comparativa intercomunitaria, la percepción de que la digitalización es positiva para el aumento de audiencia es palpable en medios en catalán, gallego e irlandés. Pero en cambio es negativa en medios en galés.

El 86\% opina que el ED en el idioma y la comunidad será positivo por la mayor visibilidad que les otorga. En cambio, el $14 \%$ opina que el ED en el idioma y la comunidad será negativo, sobre todo en comunidades que se sienten amenazadas lingüísticamente. Por comunidades, es arriesgado comparar globalmente con un número de respuestas bajo.

\section{Conclusiones}

Si después de algunos años las grandes empresas en lenguas mayoritarias no encuentran todavía un modelo de negocio fiable en el campo de los medios de comunicación en el entorno digital, en el ámbito de las lenguas menos habladas todavía resulta un reto contar con un análisis bien pautado de la situación. En el mismo The New York Times, la reducción de plantilla y el cambio de modelo de negocio a muro digital de pago es aleccionador: aún se plantea "el reto de la era digital" (The New York Times, 2013).

En el caso de los medios en las diez lenguas minoritarias europeas estudiadas, contamos con la perspectiva de sus antecedentes: la transición de la emisión analógica a la digital generó una importante incertidumbre y se dio a diferentes ritmos en cada comunidad del análisis. Con el avance de la investigación, se ha comprobado que el reto de estos medios ante el proceso de implantación digital radicaba en la financiación.

La primera conclusión destacable es la evidente perplejidad de los responsables encuestados para valorar los efectos de la digitalización y en concreto del ámbito de la financiación. Recordemos que un $40 \%$ de los responsables encuestados marca que "no sabe" cuáles son los efectos de la digitalización en la financiación. El enquistamiento de la crisis tanto económica como de los medios se agrava ante la falta de perspectivas 
de análisis y solución y de preparación financiera de los equipos ejecutivos de los medios.

Tal como hemos visto, la financiación emerge igualmente como el ámbito de los medios más afectado por la digitalización, seguida del contenido, la audiencia y el idioma, en este orden. Por un lado, los retos financieros para estas comunidades son compartidos con los de las lenguas mayoritarias (un contexto de reducción de ayudas, ventas y entradas por publicidad), pero, por otro, los problemas específicos de lenguas que conviven con otras de mayor difusión aumentan la sensación de percepción de la digitalización como un arma de doble filo que no acabará beneficiando a los pequeños.

Aunque no son mayoritarios, cabe destacar cómo la conversión digital reproduce discursos que la televisión u otras tecnologías ya habían generado; llamémosle apocalípticos. Por ejemplo, que se trata de una adaptación a la que se han visto forzados, no voluntaria, o bien es percibida como estrategia de los grandes medios para eliminar a los pequeños.

Por comunidades, hay acuerdo paneuropeo en que el efecto en la financiación es negativo, pero que es positivo en la audiencia y los contenidos. La posibilidad de llegar a más audiencia no se traduce forzosamente en un beneficio económico, por lo que ha sido correcto metodológicamente tratar en categorías distintas los efectos en diversos aspectos de la producción periodística.

En los matices que se puedan establecer entre comunidades, hay que marcar diferencias entre comunidades donde el sistema de medios, aunque ahora en crisis, está bastante normalizado (en el caso catalán o vasco), con otras comunidades muy pequeñas que se siguen sintiendo en riesgo de desaparición (galés o sami). Desde otro punto de vista, la valoración positiva sobre la financiación en el caso irlandés (tres de cada cuatro respuestas) puede darse en un contexto nacional (Irlanda) en que la economía digital ha sido muy favorecida como motor y polo de atracción de inversiones. Eso indica que, a pesar del interés novedoso de un estudio transnacional, los contextos de proximidad deben ser tenidos en cuenta por las diferentes realidades 
en políticas de comunicación, relación con las estructuras estatales y afectación de la crisis financiera y mediática.

En los medios en lenguas minoritarias, la relación con las lenguas más potentes y el tamaño reducido de gran parte de los medios agrava la dificultad de análisis y dota el contexto de conversión digital de otras especificidades, que en este estudio han sido tratadas de una manera transfronteriza y plurinacional para aportar nuevos focos al estudio de los medios en lenguas minoritarias en el mundo.

\section{Referencias}

Abramson, J. (2013). After staff reductions, new appointments at The Times. The New York Times, 29 de enero, p. B2.

Alia, V. (2010). The new media nation: Indigenous peoples and global communications. Nueva York/Oxford: Berghahn Books.

Amelina, A., Nergiz, D., Faist, T. \& Glick Schiller, N. (Eds.) (2012). Beyond methodological nationalism: Research methodologies for cross-border studies. Oxford: Routledge.

Asociación de la Prensa de Madrid (2013). Informe de la Profesión Periodística 2013. Recuperado de http://www.apmadrid.es/publicaciones/ informe-anual-de-la-profesion-periodistica. (Fecha de consulta: febrero 20 de 2014).

Browne, D. R. (1996). Electronic media and indigenous peoples: A voice of our own? Ames, Iowa: Iowa State University Press.

Casero, A. (2010). Prensa en internet: nuevos modelos de negocio en el escenario de la convergencia. El Profesional de la Información, 19(6), 595-601.

Chalmers, D., Danson, M., Lang, A. \& Milligan, L. (2013). The contribution of BBC ALBA to Gaelic: A social and economic review. En 
Jones, E. H. G. \& Uribe-Jongbloed, E. (Eds.), Social Media and minority languages: Convergence and the creative industries (212-224). Bristol: Multilingual Matters.

Collegi de Periodistes de Catalunya (2013). Dossier informe Nínxols d'ocupació. Recuperado de http://media.e-noticies.com/ext/20130220/informe---part-1.pdf (Fecha de consulta: julio 20 de 2014).

Cormack, M. \& Hourigan, N. (2007). Minority language media. Clevedon: Multilingual Matters.

Cowan, G. \& Westphal, D. (2010). Public policy and funding the news. Center on Communication Leadership \& Policy. California: USC.

Cuesta, O. (2012). Investigaciones radiofónicas: de la radio a la radio indígena. Una revisión en Colombia y Latinoamérica. Ánfora, 19(33), 165-183.

Cunliffe, D. (2009). The welsh language on the Internet. Liknguistic resistance in the age of the nertwork society. En Goggin, G. \& McLelland, M. (Eds.), Internationalizing Internet Studies: Beyond Anglophone Paradigms (96-111). Nueva York: Routledge.

Dänzler, S. (2011). Digitalization and the impact on television financing. Múnich: Nomos Publishers.

Díaz-Nosty, B. (2011). Libro negro del periodismo en España. Madrid: Fragua.

Extra, G. \& Gorter, D. (2008). Multilingual Europe: Facts and policies. Berlín: Mouton De Gruyter.

Fombona, J., Pascual, M. Á. \& Madeira, F. (2012). Realidad aumentada, una evolución de las aplicaciones de los dispositivos móviles. $P i$ xel-Bit, Revista de Medios y Educación, 41, 197-210.

Hogan-Brun, G. \& Wolff, S. (2003). Minority languages in Europe: An introduction to the current debate. En Hogan-Brun, G. y Wolff, S. 
(Eds.), Minority languages in Europe: Frameworks, status, prospects (3-15). Londres: Palgrave MacMillan.

Husband, C. (2005). Minority ethnic media as communities of practice: Professionalism and identity politics in interaction. Ethnic and $\mathrm{Mi}$ gration Studies, 31(3), 461-479.

Iriarte, M. (2006). Comunicación para el cambio social: la radio indígena comunitaria Pataxó-Ha Ha Hai. En Moneta, C. (Ed.), Jardín de senderos que se encuentran: políticas públicas y diversidad cultural en el Mercosur (117-128). Montevideo: Unesco.

Jones, E. H. G. \& Uribe-Jongbloed, E. (Eds.) (2013). Social media and minority languages: Convergence and the creative industries. Bristol: Multilingual Matters.

Kirk, J. \& Baoill, D. P. Ó. (Eds.) (2003). Towards our goals in broadcasting, the press, the performing arts and the economy: Minority Languages. En Northern Ireland, the Republic of Ireland, and Scotland. Belfast: Queens's University Belfast.

Laime, F. (2009). Radiodifusión boliviana aymara en el fortalecimiento del pueblo aymara. Más allá de las frontera (tesis de maestría). Cochabamba: Mayor de San Simón. Recuperado de http://bvirtual.proeibandes.org/bvirtual/docs/tesis/proeib/Tesis\%20Franz\%20Laime. pdf (Fecha de consulta: marzo 4 de 2014).

Lara, A. \& Vera, C. (2003). La radio en la era digital (tesis de grado). Instituto de la Comunicación y la Imagen, Universidad de Chile. Recuperado de http://www.periodismo.uchile.cl/archivos/tesis_radio. pdf (Fecha de consulta: abril 23 de 2014).

McGonagle, T. \& Moring, T. (2012). Minorities and the media: Present, probing and pressing questions. European Yearbook of Minority Issues Online, 9(1), 367-376. 
Meinhof, U.H. \& Triandafyllidou, A. (2006). Transcultural Europe: An introduction to cultural policy in a changing Europe. En Meinhof, U. H. \& Triandafyllidou, A. (Eds.), Transcultural Europe: Cultural policy in a changing Europe (3-23). Basingstoke: Palgrave Macmillan.

Nedeljković, D. V. (2011). Digitalization in Serbia in the context of media strategy of Vojvodina with focus on minority language media. $\mathrm{Me}$ dianali, 9, 57-74.

ONIC (2009). Diagnóstico integral de emisoras y/o radios indígenas. Bogotá: Ministerio de Cultura y ONIC. Recuperado de http://archivo.mintic.gov.co/mincom/documents/portal/documents/root/ mfardila/docs/DiagnosticoEmisorasIndigenas.pdf(Fecha de consulta: marzo 23 de 2014).

Oriella PR Network (2011). Estudio periodismo digital 2009-2011. Recuperado de http://www.oriellaprnetwork.com/ (Fecha de consulta: agosto 7 de 2014).

PwC (2010). Global Entertaiment and Media Outlok: 2010-2014. Resumen ejecutivo. Recuperado de http://www.pwc.es/gemo2010 (Fecha de consulta: agosto 2 de 2014).

Ramos, J. (2009). Inequidad mediática y multiculturalidad: Alcances y límites de la participación indígena en la radio oficial del Estado de Puebla, México. Congreso de la Asociación de Estudios Latinoamericanos, Río de Janeiro, Brasil, 11-14 de junio 2009. Recuperado de http: / lasa. international.pitt.edu/members/congresspapers/lasa2009/files/ RamosRodriguezJose.pdf (Fecha de consulta: enero 17 de 2014).

Riggins, S. (1992). Ethnic minority media. Londres: Sage.

Robins, K. (2006). The challenge of transcultural diversities. En Robins, K. (Ed.), The challenge of transcultural diversities: Transversal study on the theme of cultural policy and cultural diversity: Final report (1148). Estrasburgo: Consejo de Europa. 
Scolari, C. A. (2013). De las tablillas a las tablets: evolución de los e-magazines. El Profesional de la Información, 22(1), 10-18.

Starr, P. (2012). An unexpected crisis: The news media in postindustrial democracies. The International Journal of Press/Politics, 17(2), 234-242.

Taylor, P. J. (1996). On the nation-state, the global, and social science. Environment and Planning, 28, 1917-1199.

Uribe-Jongbloed, E. (2007). Una audiencia pequeña, una responsabilidad mayor: análisis del modelo europeo de creación de medios de comunicación en idiomas autóctonos minoritarios para el caso colombiano. Palabra Clave, 10(1), 48-59.

Uribe-Jongbloed, E. (2014). Minority language media studies beyond eurocentrism: Cormack's seven conditions revisited. Catalan Journal of Communication and Cultural Studies, 6(1), 35-54.

WAN-IFRA (2011).World Press Trends. Recuperado de http://www.wanifra.org/reports/2011/10/01/trends-in-newsrooms-2011 (Fecha de consulta: agosto 6 de 2014).

Wilson. P., Stewart, M. \& Córdova, A. (Eds.) (2008). Global indigenous media: Cultures, poetics, and politics. Durham/Londres: Duke University Press.

Wimmer, A. \& Glick Schiller, N. (2002). Methodological nationalism and beyond: Nation-state building, migration and the social sciences. Global Networks, 2(4), 301-334.

Wimmer, A. \& Glick Schiller, N. (2003). Methodological nationalism, the social sciences, and the study of migration: An essay in historical epistemology. International Migration Review, 37(3), 576-610. 
Zabaleta, I., Xamardo, N., Urrutia, S., Gutiérrez, A. \& Fernández, I. (2008). Language development, knowledge and use among journalists of European minority language media. Journalism Studies, 9(2), 195-211.

Zabaleta, I., Xamardo, N., Urrutia, S., Gutiérrez, A., Fernández, I. \& Ferré Pavia, C. (2010a). Between Language support and activism: A complementary journalistic function among European minoritylanguage newspeople. Journalism Studies, 11(2), 190-208.

Zabaleta, I., Xamardo, N., Gutiérrez, A., Urrutia, S. \& Fernández, I. (2010b). Transición digital y presencia en Internet de los medios de comunicación en lenguas minoritarias europeas: situación y encrucijada ante el apagón analógico. II Congreso Internacional AE-IC. Comunicación y Desarrollo en la Era Digital. Málaga, 3-5 de febrero de 2010.

Zabaleta, I., Gutiérrez, A., Ferré Pavia, C., Fernández, I., Urrutia, S. \& Xamardo, N. (2013). Website development and digital skill: The state of traditional media in European minoritylanguages. International Journal of Communication, 7, 1641-1666.

Zabaleta, I., Ferré Pavia, C., Gutiérrez, A., Fernández, I., Urrutia, S. \& Xamardo, N. (2014). European minority language media and journalism: Framing their marginal reality. International Communication Gazette, 76(3), 275-295. 